\title{
Measurements of plasma metanephrines by immunoassay vs liquid chromatography with tandem mass spectrometry for diagnosis of pheochromocytoma
}

\author{
Dirk Weismann ${ }^{1,2}$, Mirko Peitzsch ${ }^{3}$, Anna Raida ${ }^{1}$, Aleksander Prejbisz ${ }^{4}$, Maria Gosk $^{4}$, \\ Anna Riester ${ }^{5}$, Holger S Willenberg ${ }^{6}$, Reiner Klemm7, Georg Manz', \\ Timo Deutschbein ${ }^{8}$, Matthias Kroiss ${ }^{9}$, Roland Därr ${ }^{10}$, Martin Bidlingmaier ${ }^{5}$, \\ Andrzej Januszewicz ${ }^{4}$, Graeme Eisenhofer ${ }^{3,10}$ and Martin Fassnacht ${ }^{1,5,9}$ \\ ${ }^{1}$ Endocrine and Diabetes Unit, Department of Internal Medicine I, University Hospital, University of Würzburg, \\ Oberdürrbacher Str. 6, 97080 Würzburg, Germany, ${ }^{2}$ Comprehensive Heart Failure Center, University of Würzburg, \\ Würzburg, Germany, ${ }^{3}$ Institute of Clinical Chemistry and Laboratory Medicine, University Hospital Carl Gustav \\ Carus, Technische Universität Dresden, Dresden, Germany, ${ }^{4}$ Department of Hypertension, Institute of Cardiology, \\ Warsaw, Poland, ${ }^{5}$ Medizinische Klinik und Poliklinik IV, Ludwig-Maximilians-Universität München, Munich, \\ Germany, ${ }^{6}$ Division for Specific Endocrinology, Department of Endocrinology and Diabetology, Medical Faculty, \\ University Dusseldorf, Dusseldorf, Germany, ${ }^{7}$ LDN Labor Diagnostika Nord GmbH und Co. KG, Nordhorn, Germany, \\ ${ }^{8}$ Central Laboratory of the University Hospital of Wuerzburg, Würzburg, Germany, ${ }^{9}$ Comprehensive Cancer Center \\ Mainfranken, University of Würzburg, Würzburg, Germany and ${ }^{10}$ Department of Medicine III, University Hospital \\ Carl Gustav Carus, Technische Universität Dresden, Dresden, Germany
}

Correspondence should be addressed to M Fassnacht Email Fassnacht_M@ukw.de

\begin{abstract}
Background: Reports conflict concerning measurements of plasma metanephrines (MNs) for diagnosis of pheochromocytomas/paragangliomas (PPGLs) by immunoassays compared with other methods. We aimed to compare the performance of a commercially available enzyme-linked immunoassay (EIA) kit with liquid chromatography-tandem mass spectrometric (LC-MS/MS) measurements of MNs to diagnose PPGLs.

Methods: In a substudy of a prospective, multicenter trial to study the biochemical profiles of monoamine-producing tumors, we included 341 patients (174 males and 167 females) with suspected PPGLs (median age 54 years), of whom 54 had confirmed PPGLs. Plasma MNs were measured by EIA and LC-MS/MS, each in a specialized laboratory.

Results: Plasma normetanephrine (NMN) and MN were measured 60 and 39\% lower by EIA than by LC-MS/MS. Using upper cut-offs stipulated for the EIA, diagnostic sensitivity was only $74.1 \%$ at a specificity of $99.3 \%$. In contrast, use of similar cut-offs for MN and overall lower age-adjusted cut-offs for NMN measured by LC-MS/MS returned a diagnostic sensitivity and specificity of 98.1 and $99.7 \%$. Areas under receiver-operating characteristic curves, nevertheless, indicated comparable diagnostic performance of the EIA (0.993) and LC-MS/MS (0.985). Diagnostic sensitivity for the EIA increased to $96.2 \%$ with a minimal loss in specificity (95.1\%) following use of cut-offs for the EIA adapted to correct for the negative bias. Conclusions: The EIA underestimates plasma MNs and diagnostic sensitivity is poor using commonly stipulated cut-offs, resulting in a high risk for missing patients with PPGLs. Correction of this shortcoming can be achieved by appropriately determined cut-offs resulting in comparable diagnostic performance of EIA and LC-MS/MS assays.
\end{abstract}

C 2015 European Society of Endocrinology Printed in Great Britain
European Journal of Endocrinology (2015) 172, 251-260 


\section{Introduction}

The clinical presentation of pheochromocytomas/ paragangliomas (PPGLs) is highly variable, ranging from asymptomatic to life-threatening and even fatal in some cases. Autopsy studies suggest that most PPGLs are not recognized during life (1). Hypertension, either sustained or paroxysmal, is one major sign that, combined with symptoms of catecholamine excess, most usually triggers diagnostic efforts; overall, however, only a small proportion of patients tested for PPGLs are diagnosed with tumors (2). Fast and reliable methods for screening of PPGLs are required.

Biochemical diagnosis of PPGL is possible with high sensitivity and reasonable specificity using measurements of plasma free metanephrines (MNs), normetanephrine (NMN), and MN (3). Liquid chromatography with electrochemical detection (LC-ECD) was initially the method of choice for these measurements. LC-ECD has since been largely superseded by LC with tandem mass spectrometry (LC-MS/MS) (4) or immunoassay methods (5).

Numerous studies have reported high diagnostic accuracy of immunoassay measurements of plasma MNs for screening PPGLs, supporting use of the method in the routine laboratory environment $(6,7,8,9,10)$. However, none of these studies compared the immunoassay with either LC-ECD or LC-MS/MS. First reports from inter-laboratory proficiency programs suggest that immunoassays may be less precise and accurate than both other methods $(11,12)$. Suggestions that immunoassays show a negative bias, leading to potential missed diagnoses, were further suggested by two other studies $(13,14)$.

As the immunoassay has become a widely used tool for measurements of plasma MNs (especially in Europe), we compared the diagnostic performance of an enzymelinked immunoassay (EIA) with that of a recently established and validated LC-MS/MS method (15).

\section{Subjects and Methods}

The analysis took advantage of a multicenter trial (Prospective Monoamine-producing Tumor study) from which plasma samples were made available for method comparisons from 341 patients (174 males, median 54 years, range 13-86) tested for PPGLs at four European centers. The clinical protocol was approved by Local Ethics Committees at each center with written informed consent obtained from all patients.

Testing for PPGLs was carried out either because of clinical signs and symptoms (e.g. drug-resistant or paroxysmal hypertension, symptoms suggesting catecholamine excess), an incidental finding of an adrenal mass or for follow-up of patients with a history of a PPGL or a hereditary predisposition to PPGLs. Among the 341 patients tested, PPGLs were confirmed in 54 patients. Diagnosis was performed by pathological examination of surgically resected tumors, or in cases of inoperable disease, by imaging evidence of metastases. Exclusion of PPGLs in 287 patients was based on an alternative diagnosis or remission of initial complaints and an uneventful follow-up of at least 12 months.

\section{Collections of blood samples}

Blood sampling was carried out according to standard operating procedures that required an overnight fast and a supine resting position for at least $30 \mathrm{~min}$ before the blood draw. All blood samples were collected into heparinized tubes and immediately placed on ice or cool pads at $4{ }^{\circ} \mathrm{C}$. Plasma was separated by centrifugation and specimens were stored at $-80^{\circ} \mathrm{C}$ before shipping to the two central laboratories on dry ice.

\section{LC-MS/MS measurements of MNs and methoxytyramine}

In addition to measurements of $\mathrm{NMN}$ and $\mathrm{MN}$, the LC-MS/MS method allows measurements of plasma 3-methoxytyramine (3-MTY), the O-methylated metabolite of dopamine. Analyses of plasma free NMN, MN, and MTY by LC-MS/MS were performed in the Institute of Clinical Chemistry and Laboratory Medicine at the University Hospital of Dresden using a recently validated method (15). Briefly, concentrations of free NMN, MN, and 3-MTY were first purified and concentrated using an offline solid-phase extraction (SPE) procedure before injection into the LC-MS/MS. For this procedure, aliquots of samples, including calibrators and quality control samples, were loaded in a pre-conditioned SPE 96-well plate pre-filled with $10 \mathrm{mM}$ ammonium acetate and internal standard (500 pg each for deuterated NMN, deuterated MN, and deuterated MTY). Following positive pressure elution of samples, the SPE material was sequentially washed with $2 \%$ formic acid and methanol. The amine metabolites and their respective internal standards were then eluted into a 96-deep-well plate using two passes of $5 \%$ ammonia in methanol. After drying eluates in a vacuum-assisted centrifuge, residues 
were reconstituted in a solution of $2 \%$ acetonitrile and $0.2 \%$ formic acid, from which $10 \mu \mathrm{l}$ were injected into the LC-MS/MS system for analysis.

\section{Standardization and validation of the LC-MS/MS method}

LC-MS/MS measurements of NMN, MN, and MTY were performed by calibrators prepared by dilution of weighed amounts of commercially prepared material as described previously (15). Validation involved comparisons of LC-MS/MS measurements with measurements of identical samples by an established LC-ECD. That LC-ECD method in turn had shown close agreement with other methods, including comparisons of shared calibrators with other centers, and negligible bias during multiple cycles from 2008 and 2012 in an inter-laboratory quality assurance program (12).

Subsequent validations of the LC-MS/MS method included continued participation in the inter-laboratory quality assurance program, which during the 2012 cycle established minimal bias and interassay coefficient of variation $(\mathrm{CV})$ values for all three measurements of less than 5\%. Assessments of bias for the 2013 cycle indicated measurements of NMN and MN to be, respectively, $1.8 \%$ lower and 3.0\% higher than median values reported from all 27 participating laboratories, while values of 3-MTY were $8.0 \%$ lower than median values of 12 laboratories for which these measurements were reported. Proficiency program assessed interassay $\mathrm{CV}$ values to be ranging from 4.7 to $6.3 \%$ for NMN and MN and 4.4 to $11.6 \%$ for 3-MTY.

\section{EIA measurements of MNs}

EIAs for plasma free NMN and MN were performed in the laboratory of the manufacturer (Labor Diagnostica Nord (LDN), Nordhorn, Germany). The interassay CV values of the EIA range from 11.7 to $13.0 \%$ for NMN and 12.0 to $21.1 \%$ for $\mathrm{MN}$.

The EIA was established for EDTA plasma. As blood samples were collected with heparin, we validated use of heparinized plasma for the EIA using paired samples of both EDTA and heparinized plasma. Sixteen pairs of heparinized and EDTA anticoagulated samples derived from adrenal venous sampling studies were analyzed (16). Values obtained from measurements in heparinized and EDTA plasma were closely correlated for both NMN $\left(r^{2}=0.975, P<0.001\right)$ and $\mathrm{MN}\left(r^{2}=0.916, P<0.001\right)$ and showed negligible bias (Supplementary Fig. 1, see section on supplementary data given at the end of this article).
For the EIA, the lower limit of quantification (LLOQ) is $23 \mathrm{pg} / \mathrm{ml}(0.126 \mathrm{nmol} / \mathrm{l})$ for $\mathrm{NMN}$ and $17 \mathrm{pg} / \mathrm{ml}$ $(0.086 \mathrm{nmol} / \mathrm{l})$ for $\mathrm{MN}$. For LC-MS/MS, the LLOQ is $4.4 \mathrm{pg} / \mathrm{ml}(0.024 \mathrm{nmol} / \mathrm{l})$ for $\mathrm{NMN}, 3.9 \mathrm{pg} / \mathrm{ml}(0.020 \mathrm{nmol} / \mathrm{l})$ for $\mathrm{MN}$, and $4.0 \mathrm{pg} / \mathrm{ml}(0.024 \mathrm{nmol} / \mathrm{l})$ for 3-MTY.

Standardization of the EIA is achieved using mixed DL-enantiomers of NMN and MN. These concentrations are then corrected downwards to 40 and $48 \%$ of respective DL-enantiomer concentrations of NMN and $\mathrm{MN}$ to achieve estimated concentrations of L-enantiomers. Final concentrations stipulated in the instructions for use manuals reflect concentrations of L-enantiomers after a 1:6 dilution.

\section{Comparisons of LC-MS/MS and EIA measurements}

Measurements of MNs in the same samples of plasma by LC-MS/MS and EIA were performed by both laboratories in a blinded manner with no clinical information available before analyses. Comparisons also included LC-MS/MS measurements of concentrations of NMN and $\mathrm{MN}$ in calibrators used for the EIA.

\section{Data analysis}

As described previously $(15,17,18)$, upper cut-offs (UCs) for plasma free $\mathrm{NMN}$, established under supine fasting conditions, were defined by patient age, using a curvilinear regression model $\left(\mathrm{UC}_{\mathrm{NMN}}=0.0003792 \times\left(\mathrm{age}^{3}\right)+\right.$ 98.9). According to this model, UCs for NMN increased from $98.9 \mathrm{pg} / \mathrm{ml}(0.54 \mathrm{nmol} / \mathrm{l})$ for five year olds to $200 \mathrm{pg} / \mathrm{ml}(1.09 \mathrm{nmol} / \mathrm{l})$ as the maximum for all patients aged 65 years and older. The UCs were established at $88 \mathrm{pg} / \mathrm{ml}(0.45 \mathrm{nmol} / \mathrm{l})$ for $\mathrm{MN}$ and $30 \mathrm{pg} / \mathrm{ml}(0.18 \mathrm{nmol} / \mathrm{l})$ for 3-MTY.

Primarily, UCs for the immunoassay were used as published by the manufacturer $(180 \mathrm{pg} / \mathrm{ml}$ or $0.98 \mathrm{nmol} / \mathrm{l}$ for $\mathrm{NMN}$ and $90 \mathrm{pg} / \mathrm{ml}$ or $0.46 \mathrm{nmol} / \mathrm{l}$ for $\mathrm{MN}$ ). Bias of the immunoassay was quantified using Bland-Altman analysis. UCs for the EIA were then recalculated from LC-MS/MS-derived UCs to adjust for bias. We also established UCs from receiver-operating characteristic (ROC) curve analyses.

False-negative (FN) test results or true-negative results were defined as all values of both plasma free NMN and MN below their respective UCs. True-positive (TP) test results or false-positive (FP) results were defined as one or more values for plasma free NMN and MN above their respective UCs. 3-MTY was not included in the analysis unless otherwise stated. 


\section{Statistical analysis}

Continuous data are reported as medians and compared using Wilcoxon's test. Differences in diagnostic sensitivities and specificities were compared using McNemar's test and probability testing for proportions as appropriate. ROC curves were constructed based on the predicted outcomes of a multivariable logistic regression model including plasma free NMN, MN, and 3-MTY as characteristics. For analysis of diagnostic test performance, areas under the ROC curves (AUCs) were calculated and differences examined according to the method of Hanley. CIs for proportions were calculated according to the method of Newcombe (19). $P$ values of $<0.05$ were considered statistically significant. For statistical analyses, $\mathrm{R}$ version 2.12.2 (The R Foundation for Statistical Computing, Vienna, Austria) was used $(20,21)$.

\section{Results}

\section{Plasma MNs in patients with and without PPGLs}

Plasma concentrations of NMN were seven- and 8.6-fold higher in patients with PPGLs than in those without PPGLs as measured by EIA and LC-MS/MS respectively (Table 1). Plasma concentrations of MN were similarly 2.4- and 2.2-fold higher in patients with PPGLs than in those without PPGLs as measured by both EIA and LC-MS/MS. Plasma concentrations of NMN were measured to be 123 and $81 \%$ higher by LC-MS/MS than by EIA for patients with and without PPGLs respectively. Similarly, plasma concentrations of MN were measured to

Table 1 Patient characteristics and plasma concentrations of metanephrines. Values are expressed as median (range).

\begin{tabular}{|c|c|c|c|}
\hline & $\begin{array}{l}\text { Confirmed } \\
\text { PPGL }\end{array}$ & $\begin{array}{c}\text { PPGL } \\
\text { excluded }\end{array}$ & $P$ value \\
\hline$n$ & 54 & 287 & \\
\hline Age & $51.5(20-82)$ & $54.0(13-86)$ & 0.54 \\
\hline Sex (F/M) & $26 / 28$ & $141 / 146$ & 1 \\
\hline \multicolumn{4}{|l|}{ EIA } \\
\hline $\begin{array}{l}\text { Normetanephrine } \\
(\mathrm{pg} / \mathrm{ml})\end{array}$ & 224 (30-2907) & $32(22-100)$ & $<0.001$ \\
\hline $\begin{array}{l}\text { Metanephrine } \\
(\mathrm{pg} / \mathrm{ml})\end{array}$ & 39 (16-976) & $16(15-157)$ & $<0.001$ \\
\hline \multicolumn{4}{|l|}{ LC-MS/MS } \\
\hline $\begin{array}{l}\text { Normetanephrine } \\
(\mathrm{pg} / \mathrm{ml})\end{array}$ & 499 (48-9067) & $58(15-167)$ & $<0.001$ \\
\hline $\begin{array}{l}\text { Metanephrine } \\
(\mathrm{pg} / \mathrm{ml})\end{array}$ & 79 (13-2833) & $36(3-85)$ & $<0.001$ \\
\hline
\end{tabular}

$P$ values were calculated by the Mann-Whitney $U$-test. be 102 and $125 \%$ higher by LC-MS/MS than by EIA for patients with and without PPGLs.

\section{EIA vs LC-MS/MS measurements of plasma MNs}

Measurements by EIA were below LLOQs in 75 samples for plasma free NMN and 170 samples for plasma free $\mathrm{MN}$, which were therefore not included in regression or Bland-Altman analyses. With exclusion of those data, plasma concentrations of NMN and MN measured by EIA, although showing strong positive relationships $(P<0.001)$ with concentrations measured by LC-MS/MS, were measured considerably lower by EIA than by LC-MS/MS (Fig. 1). Bland-Altman plots quantified the negative bias to $60 \%$ for NMN and 39\% for MN. The negative bias was more pronounced for the higher concentrations in patients with PPGLs than for the lower concentrations in patients without tumors ( $74 \%$ vs $56 \%$ for NMN, $P<0.001$ and $52 \%$ vs $35 \%$ for $\mathrm{MN}, P=0.025)$.

\section{LC-MS/MS measurements of EIA calibrators}

Concentrations of NMN and MN measured in B, C, D, E, and $\mathrm{F}$ calibrators of the EIA were 14- to 17-fold higher than concentrations stipulated according to the instructions for use manuals of the EIA (Table 2). However, the concentrations described in those manuals reflected diluted concentrations after mixing of $100 \mu \mathrm{l}$ supplied undiluted standard solutions with $500 \mu \mathrm{l}$ equalizing solutions (i.e. 1:6 dilution). Described concentrations also reflected those of the L-enantiomers of $\mathrm{NMN}$ and $\mathrm{MN}$ calculated according to their 40 and $48 \%$ respective abundances in mixed DL-enantiomers of the starting material. With these corrections in place, concentrations measured by LC-MS/MS closely matched the concentrations of DL-NMN and -MN indicated by the manufacturers for their undiluted calibrators. For NMN, there was a near 1:1 relationship between measured concentrations and manufacturerindicated concentrations whereas, for $\mathrm{MN}$, manufacturer-indicated concentrations were $11 \%$ lower than those measured by LC-MS/MS (Supplementary Fig. 2, see section on supplementary data given at the end of this article).

\section{Diagnostic test performance}

Application of the UCs for the EIA stipulated by the manufacturers (NMN, $180 \mathrm{pg} / \mathrm{ml}$ and $\mathrm{MN}, 90 \mathrm{pg} / \mathrm{ml}$ ) resulted in a diagnostic sensitivity of $74.1 \%$ (CI, 61-83.9), 

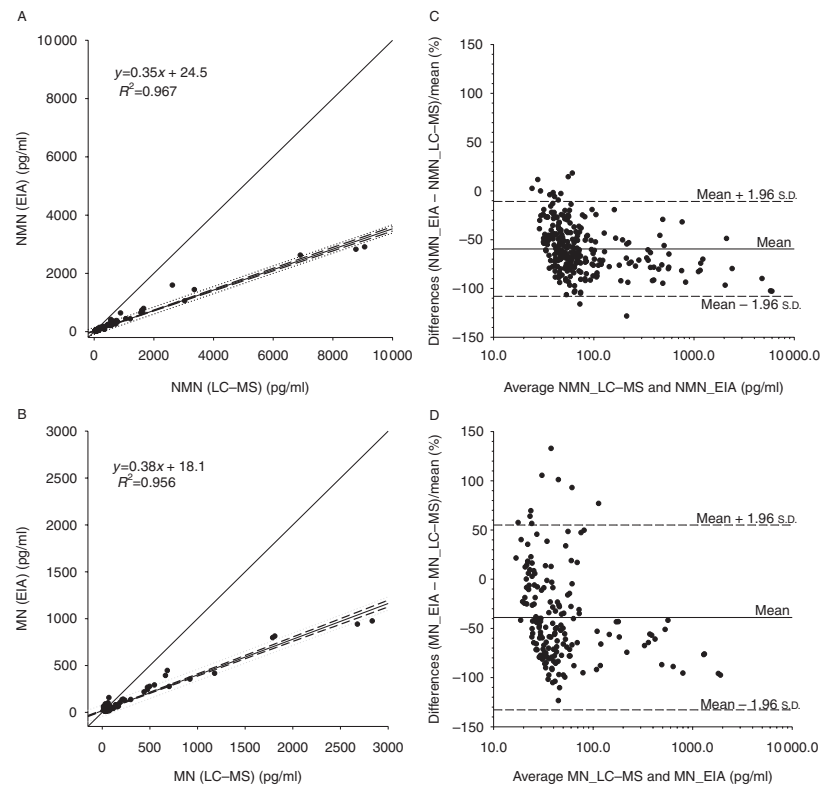

Figure 1

Comparison of NMN and MN measured by EIA and LC-MS/MS. Method of comparison for NMN (A and C) and MN (B and $D$ ) measured by EIA and LC-MS; ( $A$ and $B$ ) show the regression analysis between both methods and ( $C$ and $D$ ) show BlandAltman difference plots using the \% differences between the two methods. EIA, enzyme-linked immunoassay; LC-MS/MS, liquid chromatography with tandem mass spectrometry; NMN, plasma normetanephrine; MN, plasma metanephrine.

substantially lower $(P<0.001)$ than that of $98.1 \%$ (CI, 90.2-99.7) for measurements by LC-MS/MS (Table 3). In contrast, the diagnostic specificity did not differ between EIA- and LC-MS/MS-based measurements (98.9\% vs $99.7 \%)$. The difference in sensitivity reflected $14 \mathrm{FN}$ results using the EIA compared with only one with LC-MS/MS. The one FN result by LC-MS/MS reflected a patient with a dopamine-producing paraganglioma and metastases to the spine. Thus, when 3-MTY was combined with $\mathrm{NMN}$ and $\mathrm{MN}$, the sensitivity increased to $100 \%$ (CI, 93.4-100) with a minimal loss of specificity at $98.6 \%$ (CI, 96.5-99.5).

Despite the significantly lower diagnostic sensitivity for the EIA than for LC-MS/MS measurements, ROC curve analyses indicated AUCs that did not differ between measurements by EIA and LC-MS/MS (Fig. 2). Thus, both methods showed a comparably high diagnostic performance, indicating that the poor diagnostic sensitivity of the EIA probably reflected the use of inappropriately high UCs.
To correct for the negative bias of the EIA, UCs were adjusted downwards according to the respective 56 and $35 \%$ differences in measurements of NMN and MN by EIA and LC-MS/MS for patients without PPGLs. This yielded age-adjusted UCs for EIA measurements of NMN defined by the equation $\mathrm{UC}_{\mathrm{NMN}}=0.0001668 \times\left(\mathrm{age}^{3}\right)+43.5$. From this equation, UCs for NMN increased from $44 \mathrm{pg} / \mathrm{ml}$ $(0.24 \mathrm{nmol} / \mathrm{l})$ for five year olds up to $88 \mathrm{pg} / \mathrm{ml}$ $(0.48 \mathrm{nmol} / \mathrm{l})$ for patients 65 years and older (Table 2). Similarly, the UC for $\mathrm{MN}$ was set at $58 \mathrm{pg} / \mathrm{ml}$ $(0.294 \mathrm{nmol} / \mathrm{l})$. With these adjustments to UCs, the diagnostic sensitivity of the EIA increased to $96.2 \%$ (CI, 87.5-99) with a minimal loss in specificity to $95.1 \%$ (CI, 92-97.1). In contrast, using UCs calculated from the ROC curves $(\mathrm{NMN}, 74 \mathrm{pg} / \mathrm{ml}$ and $\mathrm{MN}, 31 \mathrm{pg} / \mathrm{ml})$, the sensitivity was $98.1 \%$ (CI, 90-99.7), and the specificity was only $87.5 \%$ (CI, 83.1-90.8).

\section{Borderline test results and biochemical phenotypes of PPGLs}

As biochemical diagnosis of PPGLs in patients with mildly elevated plasma MNs is more troublesome than that in patients with more severely elevated MNs, we also assessed the diagnostic performance of the EIA in patients with

Table 2 Immunoassay calibrator concentrations indicated by the manufacturer and measured by LC-MS/MS.

\begin{tabular}{|c|c|c|c|c|}
\hline \multirow[b]{2}{*}{ Calibrator } & \multicolumn{3}{|c|}{ EIA } & \multirow{2}{*}{$\begin{array}{c}\text { LC-MS/MS } \\
\text { DL-undiluted }\end{array}$} \\
\hline & $\begin{array}{l}\text { DL-undi- } \\
\text { luted }^{\text {a }}\end{array}$ & $\begin{array}{l}\text { L-undi- } \\
\text { luted }^{b}\end{array}$ & L-diluted ${ }^{\mathrm{c}}$ & \\
\hline \multicolumn{5}{|c|}{ NMN (pg/ml) } \\
\hline$A$ & 0 & 0 & 0 & 0 \\
\hline B & 720 & 288 & 48 & $822 \pm 56$ \\
\hline $\mathrm{C}$ & 2400 & 960 & 160 & $2688 \pm 100$ \\
\hline D & 7200 & 2880 & 480 & $8246 \pm 209$ \\
\hline$E$ & 24000 & 9600 & 1600 & $26480 \pm 626$ \\
\hline $\mathrm{F}$ & 72000 & 28800 & 4800 & $73536 \pm 1587$ \\
\hline \multicolumn{5}{|l|}{$\mathrm{MN}(\mathrm{pg} / \mathrm{ml})$} \\
\hline A & 0 & 0 & 0 & 0 \\
\hline B & 450 & 216 & 36 & $549 \pm 17$ \\
\hline $\mathrm{C}$ & 1500 & 720 & 120 & $1788 \pm 162$ \\
\hline $\mathrm{D}$ & 4500 & 2160 & 360 & $5181 \pm 200$ \\
\hline$E$ & 15000 & 7200 & 1200 & $17162 \pm 769$ \\
\hline $\mathrm{F}$ & 45000 & 21600 & 3600 & $50370 \pm 641$ \\
\hline
\end{tabular}

Three concentrations of calibrators are indicated by the manufacturer: ${ }^{a}$ DL-enantiomers of normetanephrine (NMN) and metanephrine (MN) prepared in undiluted calibrators from commercially available DL NMN and $\mathrm{MN}$; ${ }^{\mathrm{b}} \mathrm{L}$-enantiomers corrected for 40 and $48 \%$ respective proportions of L- to DL-enantiomers in commercially available DL-NMN and DL-MN 'L-enantiomers in calibrators after correction for the 1:6 dilution step (i.e. the concentrations stated in the manufacturer's instructions for use manuals). Concentrations measured by LC-MS/MS in undiluted calibrators are means \pm s.D. of three determinations for each calibrator. 
Table 3 Diagnostic test sensitivity and specificity of LC-MS/MS and EIA analytical methods.

\begin{tabular}{l} 
Method and analytes \\
\hline LC-MS/MS (without 3-MTY) \\
NMN \\
MN \\
LC-MS/MS (with 3-MTY) \\
NMN \\
MN \\
3-MTY \\
EIA (manufacturer's cut-offs) \\
NMN \\
MN \\
EIA (bias-revised cut-offs) \\
NMN \\
MN \\
EIA (ROC curve-derived cut-offs) \\
NMN \\
MN
\end{tabular}

Upper cut-offs
$99-200 \mathrm{pg} / \mathrm{ml}^{\mathrm{a}}$
$88 \mathrm{pg} / \mathrm{ml}$
$99-200 \mathrm{pg} / \mathrm{ml}^{\mathrm{a}}$
$88 \mathrm{pg} / \mathrm{ml}$
$30 \mathrm{pg} / \mathrm{ml}$
$180 \mathrm{pg} / \mathrm{ml}$
$90 \mathrm{pg} / \mathrm{ml}$
$44-88 \mathrm{pg} / \mathrm{ml}^{\mathrm{b}}$
$58 \mathrm{pg} / \mathrm{ml}$
$74 \mathrm{pg} / \mathrm{ml}$
$31 \mathrm{pg} / \mathrm{ml}$

Sensitivity \% (Cl; observations)

$98.1 \%(90.2-99.7 ; 53 / 54)$

$100 \%(93.4-100 ; 54 / 54)$

$74.1 \%(61-83.9 ; 40 / 54)$

$96.2 \%(87.5-99 ; 52 / 54)$

$98.1 \%(90-99.7 ; 53 / 54)$

NMN, normetanephrine; MN, metanephrine; 3-MTY, 3-methoxytyramine.

${ }^{a}$ Upper cut-offs for NMN (UC $\left.\mathrm{NMN}_{\mathrm{N}}\right)$ determined by an age adjustment according to the formula $\left(\mathrm{UC}_{\mathrm{NMN}}=3.792 \times 10^{-4} \times\left(\mathrm{age} \mathrm{e}^{3}\right)+98.9\right)$ from $99 \mathrm{pg} / \mathrm{ml}$ at the age of 5 years to a maximum of $200 \mathrm{pg} / \mathrm{ml}$ at the age of 65 years and above (18).

${ }^{b}$ For the EIA, age-adjusted upper cut-offs for NMN are defined by the formula $\left(\mathrm{UC}_{\mathrm{NMN}}=1.668 \times 10^{-4} \times\left(\mathrm{age} \mathrm{e}^{3}\right)+43.5\right)$ for the same age range as above.

borderline increases as defined previously (22). As shown in Fig. 3, differences between concentrations of MNs and bias-corrected upper UCs for NMN and MN as measured by EIA were smaller and less clear than differences measured by LC-MS/MS. Two patients with pheochromocytoma and multiple endocrine neoplasia type 2 (MEN2; ages 34 and 44) showed elevations in plasma MN concentrations by LC-MS/MS, but not by EIA and were thus incorrectly classified by EIA with a noradrenergic rather than an adrenergic phenotype. Moreover, with LC-MS/MS, there were 21 patients showing elevations in 3-MTY concentrations, including six with mutations in succinate dehydrogenase subunits $\mathrm{B}$ and $\mathrm{D}$ ( $S D H B$ and $S D H D$ ) genes, one of whom (age 41) had normal plasma concentrations of NMN and MN by both LC-MS/MS and EIA. Among the patients with dopamine-producing tumors, there were ten with greater elevations in 3-MTY concentrations above $80 \mathrm{pg} / \mathrm{ml}(0.48 \mathrm{nmol} / \mathrm{l})$, eight (80\%) of whom had metastatic disease. In contrast, among the other 44 patients, only three (7\%) had metastatic disease.

\section{Discussion}

This study reveals a shortcoming in a commercially available EIA kit method for measurements of plasma free MNs that can lead to a missed diagnosis in up to a quarter of patients with PPGLs. Nevertheless, ROC curve analysis demonstrated that the diagnostic performance of the EIA was comparable to that of the LC-MS/MS method, illustrating the importance of appropriately determined UCs for ensuring optimal diagnostic sensitivity and specificity. The underlying problem of substantially underestimated plasma concentrations of MNs by the EIA is compounded by use of inappropriately high UCs. As demonstrated in this study, this problem is easily addressed by use of UCs adjusted for the negative bias of EIA measurements, yielding a diagnostic sensitivity close to that of the LC-MS/MS method with a minimal loss in specificity.

The negative bias of EIA measurements of plasma MNs was initially suggested from results of an inter-laboratory quality assurance program that utilized pooled lyophilized plasma spiked with commercially available racemic enantiomers of MNs in the first series (11), and plasma spiked with natural L-enantiomers derived from urine was used in a subsequent series (12). The distinction is important, as the natural L-enantiomers of $\mathrm{MN}$ are the forms targeted by immunoassays (5). In contrast, chromatographic methods do not distinguish between the $\mathrm{D}$ - and L-enantiomers, allowing the use of commercially available racemic enantiomers for calibration of those methods.

In this study, we explored the above issues further by LC-MS/MS measurements of NMN and MN in EIA calibrators, observing close agreement between measured concentrations and those indicated by the manufacturer. These expected concentrations were, however, those of the DL MNs that the manufacturer uses for preparation of standards, with final lower concentrations in instruction 
A

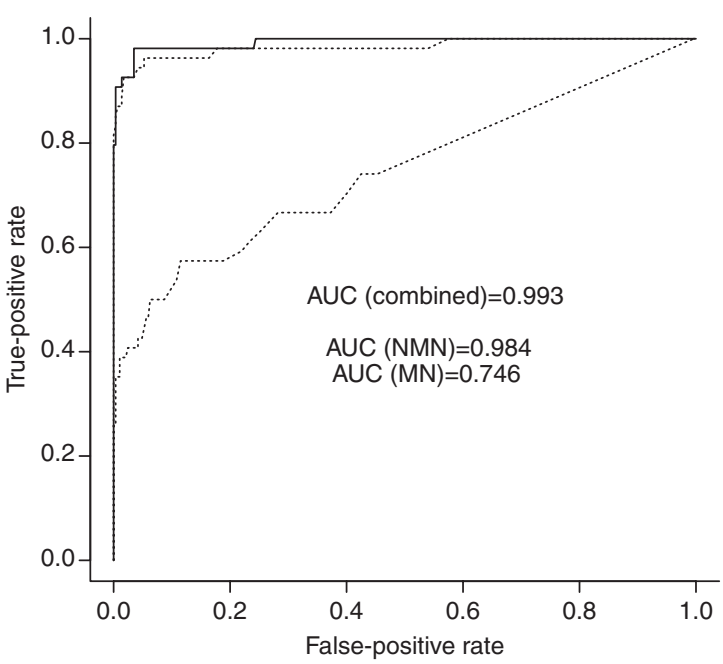

B

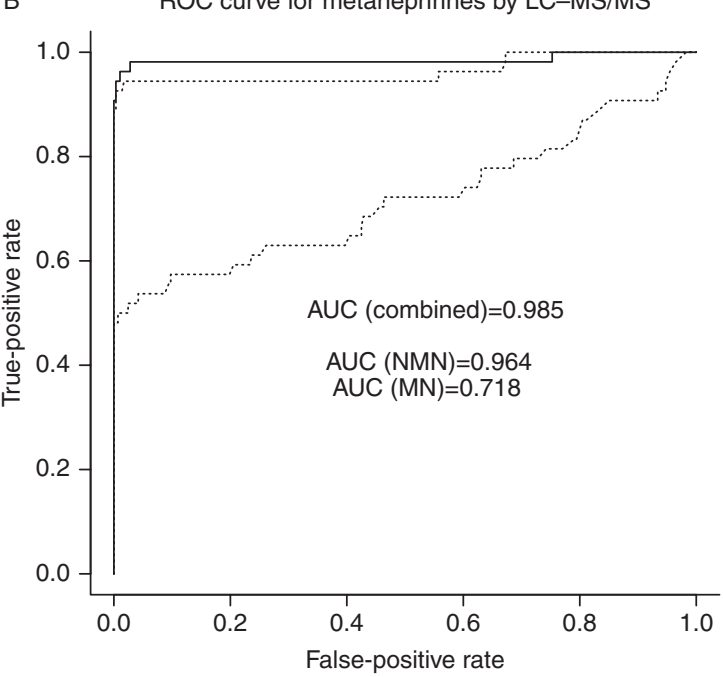

C

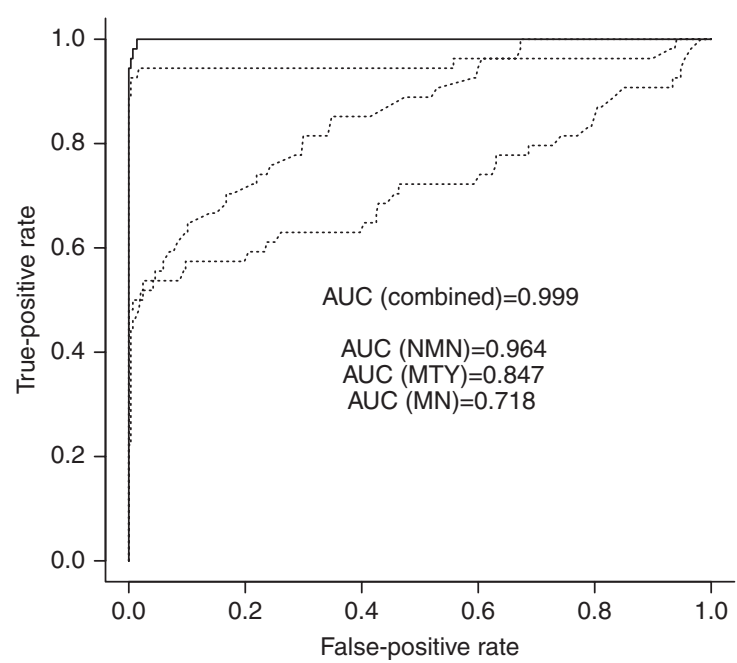

for use manuals reflecting estimated concentrations of L-enantiomers. Therefore, it seems possible that the DL- to L-enantiomer correction may represent the cause of the lower concentrations of MNs measured by the EIA than by LC-MS/MS.

The ideal solution to the EIA calibration problem would be met by use of L-enantiomers of MNs, which, unfortunately, are presently unavailable from commercial suppliers. In the meantime, miscalibration of the EIA combined with inappropriately used reference intervals has already led to reports of patients with PPGLs being missed by the EIA that were detected by LC-MS/MS. In the report by Peaston et al. (14), two patients with PPGLs were described with repeatedly negative results by EIA and positive results by LC-MS/MS. In this and previous reports $(13,14)$, concerns were raised about the likelihood of FN results for the EIA according to the UCs indicated by the manufacturer. The findings of this study in a larger series of patients prospectively tested for PPGLs now confirm that these concerns are justified.

While we now confirm that the UCs provided by the manufacturer are inappropriately high, other users of commercial immunoassays for plasma MNs have appropriately determined their own UCs $(10,23)$. Using ROC curve analysis, Unger et al. established UCs for an early RIA much lower than that stipulated by the manufacturers resulting in a diagnostic sensitivity of $95.8 \%$, but at a specificity of only $79.4 \%$. We similarly established a specificity less than the optimal specificity using ROC curve-derived UCs, supporting use of the bias-corrected UCs described in this study. More recently, Pussard et al. (23), using a RIA kit from a different manufacturer, have reported that the UCs stipulated for that kit were also too high and described optimal UCs for NMN to be $100 \mathrm{pg} / \mathrm{ml}$

\section{Figure 2}

ROC curve analysis for plasma free NMN, MN, and 3-methoxytyramine (if applicable) by EIA and LC-MS/MS. EIA, enzyme-linked immunoassay; LC-MS/MS, liquid chromatography with tandem mass spectrometry; NMN, plasma normetanephrine; MN, plasma metanephrine; ROC, receiver-operating characteristics; AUC, area under the curve. ROC curves for comparison of test performance of plasma MNs measured by EIA (A) and LC-MS/MS (B). Results including 3-methoxytyramine are shown for LC-MS/MS (C). The solid line shows the test characteristics of combined analysis of NMN and $\mathrm{MN}$ on the diagnostic performance. Dashed lines give the characteristics of either NMN or MN. 


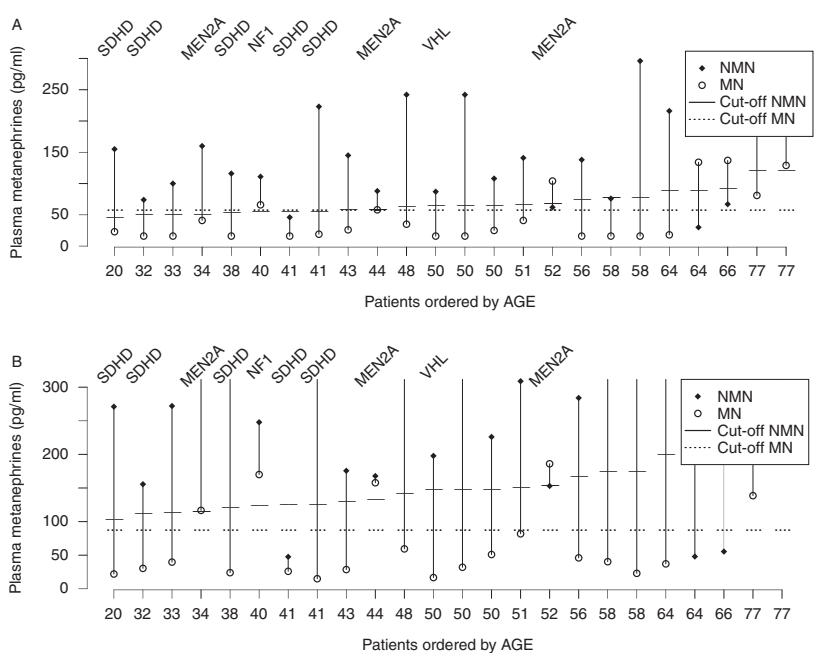

\section{Figure 3}

Plasma free NMN and MN results by EIA (top) and LC-MS/MS (bottom) in patients with borderline results. EIA, enzyme-linked immunoassay; LC-MS/MS, liquid chromatography with tandem mass spectrometry; NMN, plasma normetanephrine; MN, plasma metanephrine; MEN2A, multiple endocrine neoplasia type 2A; NF1, neurofibromatosis type 1; VHL, von HippelLindau; SDHB, succinate dehydrogenase B; SDHD, succinate dehydrogenase $\mathrm{D}$. Results for $\mathrm{NMN}$ and $\mathrm{MN}$ in individual patients with moderately increased NMN and MN values are shown as measured by EIA (A) and LC-MS/MS (B). Patients are ordered by age. UCs for NMN (age-adjusted) and MN (static) are given. Hereditary diseases are specified, if applicable.

$(0.55 \mathrm{nmol} / \mathrm{l})$ and $\mathrm{MN}$ to be $70 \mathrm{pg} / \mathrm{ml}(0.36 \mathrm{nmol} / \mathrm{l})$. However, by applying these UCs to our series, diagnostic sensitivity was only increased from 74 to $90 \%$.

Rather than relying on information provided by manufacturers of kits, UCs should be verified in each laboratory according to published guidelines (24). Ideally, reference intervals should also be further validated with appropriately sized populations of patients with and without disease. For manufacturers of diagnostic kits for rare endocrine diseases, such validation and continued quality assurance can pose a problem due to limited access to patient materials. Closer interactions between kit manufacturers and high-patient-volume academic reference centers may provide a partial solution. For lower volume laboratories that utilize such kits, a practical approach might be local verification of calculations from larger series, such as this study. Continuous local validation by participation in inter-laboratory quality assurance programs is also essential.
As has been demonstrated in a recent report (17), it should be appreciated that use of inappropriately high UCs for measurements of plasma MNs is not simply a problem confined to commercial immunoassay kits, but also a problem for laboratories where UCs have been established from reference populations sampled in the seated position rather than the recommended supine position. As indicated in that report, use of UCs established in the seated position results in a substantially decreased diagnostic sensitivity associated with a ninefold increase in FN results. Again, this illustrates the crucial importance of correctly determined UCs to effective diagnosis of PPGLs.

While use of the EIA with UCs provided in this study results in an acceptable diagnostic sensitivity, we also show that careful interpretation of borderline-increased results is particularly important as measurements by the EIA are much closer to the UCs compared with those with LC-MS/MS. As plasma concentrations of MNs correlate with the tumor size (25), generally special attention must be given to smaller tumors with borderline-increased biochemical results (26). Thus, patients monitored after resection of a PPGL will usually have much lower plasma concentrations of MNs in the early stage of a relapse, irrespective of a malignant course, a hereditary disease, or a sporadic relapse (27).

In our study, the proportion of patients with PPGLs to those without disease was higher than expected for the usual pre-test prevalence of the tumor. Among unselected patients screened for PPGLs, prevalence of PPGLs ranges from 0.8 to $1.6 \%(28,29,30)$. Among patients with incidentalomas, prevalence of PPGLs is higher, at 4-9\% (31), but even higher for patients tested because of germline mutations of PPGL susceptibility genes, reaching to $40 \%$ in patients with MEN2 (32). Similarly, patients with a history of PPGLs have an overall 16.5\% risk of recurrent disease (27). For all above groups of patients, UCs should be chosen to ensure a high diagnostic sensitivity. While such UCs have a minimal impact on relative proportions of FP results to TP results for testing among high-risk groups, the impact becomes larger at low prevalence rates. This may favor LC-MS/MS over immunoassays for minimizing rates of FP results when testing low-risk groups.

LC-MS/MS offers several other advantages over EIA assays. While immunoassays are relatively easy to implement, matrix effects are well-recognized limitations of this method, resulting in cross-reactivity, nonspecific binding, or interference; inter- and intra-assay variabilities may also cause difficulties for patient follow-up (33). 
LC-ECD can also suffer from interferences, whereas LC-MS/MS provides greater freedom from analytical interference (4). Nevertheless, some interferences with LC-MS/MS have been reported due to isobaric compounds (compounds with the same element composition) for which precursor and product ions may not be distinguished by collision-induced dissociation (34). Such interferences, however, can be easily avoided by chromatographic separation. Finally, the addition of 3-MTY to the diagnostic work-up further increases sensitivity with only a marginal loss in specificity (Table 3). Nevertheless, such high sensitivities and specificities can only be attained by supine and fasting sampling (17).

As measurements of 3-MTY are not currently available for EIA, it is also not yet possible using the EIA to detect PPGLs that produce only dopamine or correctly classify PPGLs with dopaminergic phenotypes. Thus, one patient with a SDHD mutation and a purely dopamine-secreting tumor was missed by the EIA, but detected by LC-MS/MS. Metastatic disease in several others was identified by measurements of 3-MTY. Furthermore, two patients with MEN2A were falsely diagnosed by EIA with norepinephrine phenotypic features. As indicated by the numbers of results below LLOQs, analytical sensitivity of the EIA is less than that provided by LC-MS/MS. Catecholamine profiling can assist with identifying underlying mutations and presence of malignant disease, but, for this, analytical sensitivity sufficient for precise measurements of all three metabolites at the lower end of the scale is essential $(35,36)$.

In conclusion, ROC curve analysis shows that both LC-MS/MS and EIA measurements of plasma MNs provide excellent diagnostic performance in patients tested for PPGLs. However, the EIA measures the metabolites to be very low, hence re-calibration of the method is needed. More importantly, cut-offs indicated by the manufacturer do not provide sufficient sensitivity for diagnosis of PPGLs. Until the calibration problem is corrected, this shortcoming can be overcome using UCs adjusted for the negative bias as defined in this study.

\section{Supplementary data}

This is linked to the online version of the paper at http://dx.doi.org/10.1530/ EJE-14-0730.

\section{Declaration of interest}

R Klemm and G Manz are employees of LDN (Nordhorn, Germany). The other authors have nothing to disclose.

\section{Funding}

This work was supported by the Deutsche Forschungsgemeinschaft (KFO252; El855/1-1) and the European Union Seventh Framework Programme (FP7/2007-2013) under grant agreement 259735 (ENS@TCancer) as well as the Institute of Cardiology, Warsaw, Poland (grant number 2.21/VII/12).

\section{References}

1 Sutton MG, Sheps SG \& Lie JT. Prevalence of clinically unsuspected pheochromocytoma. Review of a 50-year autopsy series. Mayo Clinic Proceedings 198156 354-360.

2 Chen H, Sippel RS, O’Dorisio MS, Vinik AI, Lloyd RV \& Pacak K. The North American Neuroendocrine Tumor Society consensus guideline for the diagnosis and management of neuroendocrine tumors: pheochromocytoma, paraganglioma, and medullary thyroid cancer. Pancreas 201039 775-783. (doi:10.1097/MPA.0b013e3181ebb4f0)

3 Lenders JW, Pacak K, Walther MM, Linehan WM, Mannelli M, Friberg P, Keiser HR, Goldstein DS \& Eisenhofer G. Biochemical diagnosis of pheochromocytoma: which test is best? Journal of the American Medical Association 2002287 1427-1434. (doi:10.1001/jama.287.11. 1427)

4 Grebe SK \& Singh RJ. LC-MS/MS in the clinical laboratory - where to from here? Clinical Biochemist. Reviews 201132 5-31.

5 Manz B, Kuper M, Booltink E \& Fischer-Brugge U. Development of enantioselective immunoassays for free plasma metanephrines. Annals of the New York Academy of Sciences 20041018 582-587. (doi:10.1196/ annals.1296.072)

6 Christensen TT, Frystyk J \& Poulsen PL. Comparison of plasma metanephrines measured by a commercial immunoassay and urinary catecholamines in the diagnosis of pheochromocytoma. Scandinavian Journal of Clinical and Laboratory Investigation 201171 695-700. (doi:10.3109/00365513.2011.622410)

7 Gao YC, Lu HK, Luo QY, Chen LB, Ding Y \& Zhu RS. Comparison of free plasma metanephrines enzyme immunoassay with (131)I-MIBG scan in diagnosis of pheochromocytoma. Clinical and Experimental Medicine 20088 87-91. (doi:10.1007/s10238-008-0162-2)

8 Procopiou M, Finney H, Akker SA, Chew SL, Drake WM, Burrin J \& Grossman AB. Evaluation of an enzyme immunoassay for plasma-free metanephrines in the diagnosis of catecholamine-secreting tumors. European Journal of Endocrinology 2009161 131-140. (doi:10.1530/ EJE-09-0172)

9 Sarathi V, Pandit R, Jagtap V, Lila AR, Bandgar TR, Menon PS, Varthakavi P, Raghavan VP \& Shah NS. Performance of plasma fractionated free metanephrines by enzyme immunoassay in the diagnosis of pheochromocytoma and paraganglioma. Endocrine Practice 201117 759-765. (doi:10.4158/EP11058.OR)

10 Unger N, Pitt C, Schmidt IL, Walz MK, Schmid KW, Philipp T, Mann K $\&$ Petersenn S. Diagnostic value of various biochemical parameters for the diagnosis of pheochromocytoma in patients with adrenal mass. European Journal of Endocrinology 2006 154 409-417. (doi:10.1530/ eje.1.02097)

11 Pillai D, Ross HA, Kratzsch J, Pedrosa W, Kema I, Hoad K, Rouaix N, Fathi M, Nader H, Mathian B et al. Proficiency test of plasma free and total metanephrines: report from a study group. Clinical Chemistry and Laboratory Medicine 200947 786-790. (doi:10.1515/CCLM.2009.162)

12 Pillai D \& Callen S. Pilot quality assurance programme for plasma metanephrines. Annals of Clinical Biochemistry 201047 137-142. (doi:10.1258/acb.2009.009153)

13 Mullins F, O'Shea P, FitzGerald R \& Tormey W. Enzyme-linked immunoassay for plasma-free metanephrines in the biochemical diagnosis of phaeochromocytoma in adults is not ideal. Clinical 
Chemistry and Laboratory Medicine 201250 105-110. (doi:10.1515/ cclm.2011.742)

14 Peaston RT, Graham KS, Chambers E, van der Molen JC \& Ball S. Performance of plasma free metanephrines measured by liquid chromatography-tandem mass spectrometry in the diagnosis of pheochromocytoma. Clinica Chimica Acta 2010411 546-552. (doi:10.1016/j.cca.2010.01.012)

15 Peitzsch M, Prejbisz A, Kroiss M, Beuschlein F, Arlt W, Januszewicz A, Siegert G \& Eisenhofer G. Analysis of plasma 3-methoxytyramine, normetanephrine and metanephrine by ultraperformance liquid chromatography-tandem mass spectrometry: utility for diagnosis of dopamine-producing metastatic phaeochromocytoma. Annals of Clinical Biochemistry 201350 147-155. (doi:10.1258/acb.2012.012112)

16 Dekkers T, Deinum J, Schultzekool LJ, Blondin D, Vonend O, Hermus AR, Peitzsch M, Rump LC, Antoch G, Sweep FC et al. Plasma metanephrine for assessing the selectivity of adrenal venous sampling. Hypertension 201362 1152-1157. (doi:10.1161/HYPERTENSIONAHA. 113.01601)

17 Darr R, Pamporaki C, Peitzsch M, Miehle K, Prejbisz A, Peczkowska M, Weismann D, Beuschlein F, Sinnott R, Bornstein SR et al. Biochemical diagnosis of phaeochromocytoma using plasma-free normetanephrine, metanephrine and methoxytyramine: importance of supine sampling under fasting conditions. Clinical Endocrinology 201480 478-486. (doi:10.1111/cen.12327)

18 Eisenhofer G, Lattke P, Herberg M, Siegert G, Qin N, Darr R, Hoyer J, Villringer A, Prejbisz A, Januszewicz A et al. Reference intervals for plasma free metanephrines with an age adjustment for normetanephrine for optimized laboratory testing of phaeochromocytoma. Annals of Clinical Biochemistry 201350 62-69. (doi:10.1258/acb.2012. 012066)

19 Newcombe RG. Two-sided confidence intervals for the single proportion: comparison of seven methods. Statistics in Medicine $1998 \mathbf{1 7}$ 857-872. (doi:10.1002/(SICI)1097-0258(19980430)17:8 <857::AIDSIM777 > 3.0.CO;2-E)

20 Sing T, Sander O, Beerenwinkel N \& Lengauer T. ROCR: visualizing classifier performance in R. Bioinformatics 200521 3940-3941. (doi:10.1093/bioinformatics/bti623)

21 R Core Team. R: A language and environment for statistical computing. R Foundation for Statistical Computing, Vienna, Austria. 2013. http://www.R-project.org/.

22 Lenders JW, Eisenhofer G, Mannelli M \& Pacak K. Phaeochromocytoma. Lancet 2005366 665-675. (doi:10.1016/S0140-6736(05)67139-5)

23 Pussard E, Chaouch A \& Said T. Radioimmunoassay of free plasma metanephrines for the diagnosis of catecholamine-producing tumors. Clinical Chemistry and Laboratory Medicine 201452 437-444. (doi:10.1515/cclm-2013-0406)

24 CLSI. Defining, establishing, and verifying reference intervals in the clinical laboratory; approved guideline. Third edition. In CLSI Document, EP28-A3: Wayne, PA: Clinical and Laboratory Standards Institute, 2010.
25 Eisenhofer G, Lenders JW, Goldstein DS, Mannelli M, Csako G, Walther MM, Brouwers FM \& Pacak K. Pheochromocytoma catecholamine phenotypes and prediction of tumor size and location by use of plasma free metanephrines. Clinical Chemistry $2005 \mathbf{5 1}$ 735-744. (doi:10.1373/clinchem.2004.045484)

26 Anas SS \& Vasikaran SD. An audit of management of patients with borderline increased plasma-free metanephrines. Annals of Clinical Biochemistry 201047 554-558. (doi:10.1258/acb.2010.010131)

27 Amar L, Fassnacht M, Gimenez-Roqueplo AP, Januszewicz A, Prejbisz A, Timmers H \& Plouin PF. Long-term postoperative follow-up in patients with apparently benign pheochromocytoma and paraganglioma. Hormone and Metabolic Research 201244 385-389. (doi:10.1055/ s-0031-1301339)

28 Brain KL, Kay J \& Shine B. Measurement of urinary metanephrines to screen for pheochromocytoma in an unselected hospital referral population. Clinical Chemistry 200652 2060-2064. (doi:10.1373/ clinchem.2006.070805)

29 Hernandez FC, Sanchez M, Alvarez A, Diaz J, Pascual R, Perez M, Tovar I $\&$ Martinez P. A five-year report on experience in the detection of pheochromocytoma. Clinical Biochemistry 200033 649-655. (doi:10.1016/S0009-9120(00)00172-7)

30 Vaclavik J, Stejskal D, Lacnak B, Lazarova M, Jedelsky L, Kadalova L, Janosova M, Frysak Z \& Vlcek P. Free plasma metanephrines as a screening test for pheochromocytoma in low-risk patients. Journal of Hypertension 200725 1427-1431. (doi:10.1097/HJH. 0b013e32813aeb5a)

31 Terzolo M, Bovio S, Pia A, Reimondo G \& Angeli A. Management of adrenal incidentaloma. Best Practice \& Research. Clinical Endocrinology \& Metabolism 200923 233-243. (doi:10.1016/j.beem.2009.04.001)

32 Howe JR, Norton JA \& Wells SA Jr. Prevalence of pheochromocytoma and hyperparathyroidism in multiple endocrine neoplasia type 2A: results of long-term follow-up. Surgery 1993114 1070-1077.

33 de Jong WH, de Vries EG \& Kema IP. Current status and future developments of LC-MS/MS in clinical chemistry for quantification of biogenic amines. Clinical Biochemistry 201144 95-103. (doi:10.1016/ j.clinbiochem.2010.07.006)

34 Twentyman JM, Cradic KW, Singh RJ \& Grebe SK. Ionic cross talk can lead to overestimation of 3-methoxytyramine during quantification of metanephrines by mass spectrometry. Clinical Chemistry $2012 \mathbf{5 8}$ 1156-1158. (doi:10.1373/clinchem.2012.186601)

35 Bausch B, Koschker AC, Fassnacht M, Stoevesandt J, Hoffmann MM, Eng C, Allolio B \& Neumann HP. Comprehensive mutation scanning of NF1 in apparently sporadic cases of pheochromocytoma. Journal of Clinical Endocrinology and Metabolism 200691 3478-3481. (doi:10.1210/ jc.2006-0780)

36 Eisenhofer G, Lenders JW, Timmers H, Mannelli M, Grebe SK, Hofbauer LC, Bornstein SR, Tiebel O, Adams K, Bratslavsky G et al. Measurements of plasma methoxytyramine, normetanephrine, and metanephrine as discriminators of different hereditary forms of pheochromocytoma. Clinical Chemistry 201157 411-420. (doi:10.1373/clinchem.2010.153320)

Received 27 August 2014

Revised version received 1 November 2014

Accepted 28 November 2014 